INPLASY

PROTOCOL

To cite: Ping et al. Can acupuncture improve sleep quality and anxiety among women during perimenopause?A protocol for systemic review and metaanalysis. Inplasy protocol 2021120012. doi: 10.37766/inplasy2021.12.0012

Received: 02 December 2021

Published: 02 December 2021

Corresponding author:

Zhongnan Wang

287695427@qq.com

Author Affiliation: Changchun University of Chinese Medicine.

Support: JL ISAT

150414045GH.

Review Stage at time of this submission: Preliminary searches.

Conflicts of interest:

None declared.

\section{Can acupuncture improve sleep quality and anxiety among women during perimenopause?A protocol for systemic review and meta-analysis}

Ping, YP1; Liang, C2; Fan, XX33 Zhang, LL4; Ying, DS5; Wang, ZN6.

Review question / Objective: The decrease in estrogen levels during the perimenopausal period can cause women to have various symptoms such as insomnia, emotional anxiety and even depression.Therefore, whether the green therapy of acupuncture can improve the sleep quality and anxiety of perimenopausal women has attracted more and more attention. The purpose of this systematic review is to evaluate the efficacy of acupuncture. P: Perimenopausal women with insomnia and anxiety; I: Acupuncture therapy (traditional acupuncture and electro-acupuncture therapy); C: Drug therapy, Chinese herbal medicine, music therapy, cognitive behavioral therapy (CBI) and placebo; O: Effective rate, PSQI, Hamilton Anxiety Scale (HAMA) and Hamilton Depression Scale (HAMD); S: RCT.

INPLASY registration number: This protocol was registered with the International Platform of Registered Systematic Review and Meta-Analysis Protocols (INPLASY) on 02 December 2021 and was last updated on 02 December 2021 (registration number INPLASY2021120012).

\section{INTRODUCTION}

Review question / Objective: The decrease in estrogen levels during the perimenopausal period can cause women to have various symptoms such as insomnia, emotional anxiety and even
depression.Therefore, whether the green therapy of acupuncture can improve the sleep quality and anxiety of perimenopausal women has attracted more and more attention. The purpose of this systematic review is to evaluate the efficacy of acupuncture. P: Perimenopausal 
women with insomnia and anxiety; I: Acupuncture therapy (traditional acupuncture and electro-acupuncture therapy); C: Drug therapy, Chinese herbal medicine, music therapy, cognitive behavioral therapy (CBI) and placebo; $O$ : Effective rate, PSQI, Hamilton Anxiety Scale (HAMA) and Hamilton Depression Scale (HAMD); S: RCT.

Condition being studied: Insomnia and emotional anxiety are common health problems in perimenopausal women.More than $50 \%$ of women have problems starting or maintaining sleep. Chronic insomnia and anxiety are also considered to be risk factors that contribute to medical problems such as cardiovascular disease, diabetes, obesity and asthma, which emphasizes the necessity of identifying and treating insomnia.It is not surprising that there is universal interest in finding effective complementary and alternative therapies to treat insomnia and anxiety with a lower risk of adverse effects or withdrawal.Therefore, the purpose of this study was to systematically evaluate the efficacy and safety of acupuncture in the treatment of insomnia and anxiety in perimenopausal women.

\section{METHODS}

Participant or population: Perimenopausal women are clearly identified by clinicians as chronic sleeplessness accompanied by emotional anxiety.

Intervention: The intervention treatment group included traditional acupuncture, warm acupuncture and electric acupuncture.

Comparator: The control group included Drug therapy, Chinese herbal medicine, music therapy, cognitive-behavioral therapy $(\mathrm{CBI})$ and placebo.

Study designs to be included: All randomized controlled trials on the treatment of insomnia and anxiety in perimenopausal women with acupuncture and moxibustion will be included in this study.
Eligibility criteria: Inclusion criteria.1. The type of study must be a randomized controlled trial of clinical observation. 2. Participants must be perimenopausal women with a clinical diagnosis of insomnia and depression. 3. Patients without severe underlying cardiac, hepatic or renal disease. Exclusion criteria.1. literature with very small sample sizes had to be excluded. 2. Patients underwent other relevant treatment prior to the intervention. 3. This study did not include systematic evaluation, review and animal experiments. 4. literature with poor experimental design, erroneous data or unextractable data is not eligible for this study.

Information sources: We will search for clinical observational pilot studies of acupuncture for insomnia, anxiety or depression included in PubMed, Cochrane Library , Embase, Web of science, China Knowledge Network (CNKI), Wanfang, VIP and China Biomedical Database (CBM), etc. The search period will be from the establishment of the database until November 2021.

Main outcome(s): The total clinical effectiveness rate was used to observe the efficacy of acupuncture in treating insomnia and depression, and the total clinical effectiveness rate was used as the primary outcome index.

Additional outcome(s): The Pittsburgh Sleep Quality Index (PSQI) was used to assess the sleep quality of perimenopausal women. Hamilton Anxiety Inventory (HAMA) and Hamilton Depression Inventory (HAMD) were used to assess patients' anxiety and depression indices.

Quality assessment / Risk of bias analysis: We will use RevMan software to evaluate the quality of the literature by the Cochrane risk bias assessment tool for six aspects of the included literature, including random assignment method, allocation scheme concealment, blinding, completeness of outcome data, selective reporting of study results, and other sources of bias, and the evaluation levels are divided into three 
levels: low risk bias, high risk bias, and unclear.

Strategy of data synthesis: First, the included literature was tested for heterogeneity, and when $P \geq 0.1$ and $I 2<$ $50 \%$ suggested no significant statistical heterogeneity, a fixed-effects model was used; when $P<0.1$ and $I 2 \geq 50 \%$ suggested the existence of statistical heterogeneity, if the degree of heterogeneity was within an acceptable range, a random-effects model was selected for the combined analysis, while subgroup analysis, sensitivity analysis and Meta-regression should be performed according to the situation to explain the source of heterogeneity. The dichotomous variables were analyzed statistically using relative risk (RR); the continuous variables were analyzed using mean standard deviation (MD) or weighted (SMD) as the combined effect measure, and their $95 \%$ confidence intervals (Cl) were calculated.

Subgroup analysis: In case of significant heterogeneity, we will conduct subgroup studies based on the type of insomnia, the age of the patient, and the intervention.

Sensitivity analysis: When the combined results of the remaining documents were not significantly different from those without deletion after the deletion of any one of them, it means that the sensitivity analysis was passed.

Country(ies) involved: China.

Keywords: Acupuncture, sleep, anxiety, Perimenopause, systematic review.

Contributions of each author:

Author 1 - Yanpei Ping.

Author 2 - Chao Liang.

Author 3 - Xixi Fan.

Author 4 - Lili Zhang.

Author 5 - Dashi Ying.

Author 6 - Zhongnan Wang. 